\title{
RISK ANALYSIS OF COMBINED RAINWATER DETENTION AND PUMPING SYSTEM
}

\author{
Nadia Ursino \\ Department ICEA, University of Padova, Padova, Italy. \\ Email: nadia.ursino@unipd.it
}

April 8, 2016

\section{Supplemetary Material. Analytical deriva- tion of Risk Models}

In a conceptualized SSS, detention is achieved by a storage capacity (SU) and a pumping station (PS) ensures flow continuity to the final destination, because gravity drainage is not possible (Figure 1). Two set-ups are examined here: above-ground SU downstream of the PS (Figure 1a) and SU upstream of it (Figure $1 b$ ).

\subsection{Risk of overflow of combined PS and above-ground SU}

Assuming that, when rain begins to fall, the PS discharge capacity is $i Q_{p}$ because $n+r-i$ pumps have failed, the probability that the inflow discharge to the $\mathrm{SU}$ exceeds the pumps discharge capacity $\left(\varphi S h \tau^{-1}>i Q_{p}\right)$ is:

$$
R_{1 a}=\int_{0}^{\infty} f_{h} \int_{0}^{\frac{\varphi S h}{i Q_{p}}} f_{\tau} d \tau d h=\int_{0}^{\infty} f_{\tau} \int_{\frac{i Q_{p} \tau}{\varphi S h}}^{\infty} f_{h} d h d \tau
$$

Although $\tau>\varphi S h\left(i Q_{p}\right)^{-1}$ or equivalently $h<i Q_{p} \tau(\varphi S)^{-1}$, so that no failure occurs at the PS, the system shown in Figure 1a, may overflow 


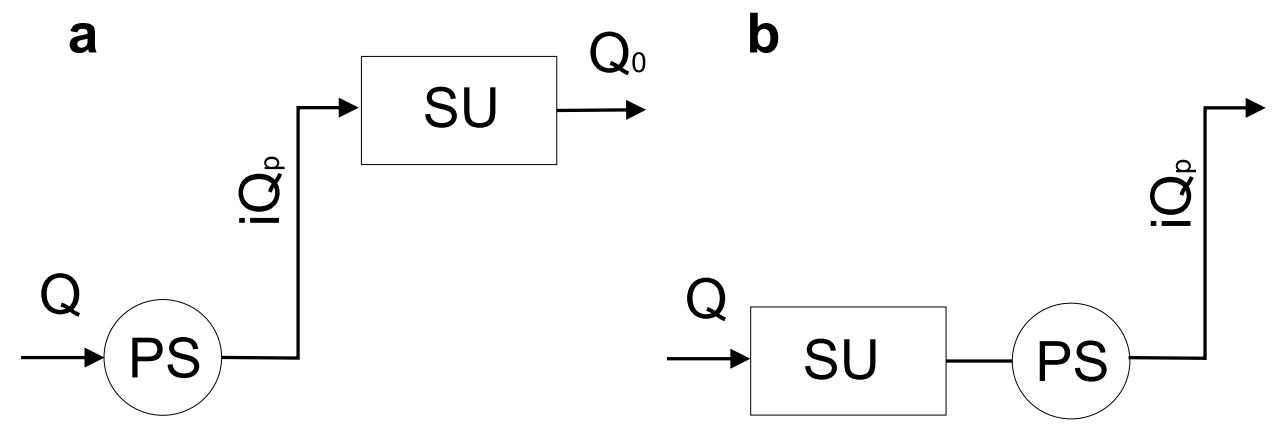

Figure 1: Conceptual model of low impact structural measures for storm sewer system (SSS). SU =storage unit, $\mathrm{PS}=$ pumping station.

even at SU. Assuming that the SU is full at the end of the first of two consecutive rain events, if their inter-arrival time is: $t>V_{s} \cdot\left(Q_{0}\right)^{-1}$, the $\mathrm{SU}$ is empty before the second rainfall event and overflow occurs if $i Q_{p} \tau>V_{s}$. Conversely, if $t<V_{s} \cdot\left(Q_{0}\right)^{-1}$, the residual storage capacity available for flood control is $t \cdot Q_{0}<V_{s}$ and overflow occurs if $i Q_{p} \tau>Q_{0} t$. Thus, the risk of $\mathrm{SU}$ overflow, provided that the PS did not undergo any $\left(Q<i Q_{p}\right)$ is:

$$
\begin{array}{r}
R_{2 a}(i)=\int_{I E T D}^{\frac{V_{s}}{Q_{0}}} f_{t} \int_{\frac{Q_{0} t}{i Q_{p}}}^{\infty} f_{\tau} \int_{0}^{\frac{i Q_{p} \tau}{\varphi S}} f_{h} d h d \tau d t+ \\
\int_{\frac{V_{s}}{Q_{0}}}^{\infty} f_{t} \int_{\frac{V_{s}}{i Q_{p}}}^{\infty} f_{\tau} \int_{0}^{\frac{i Q_{p} \tau}{\varphi S}} f_{h} d h d \tau d t
\end{array}
$$

$R_{1 a}$ and $R_{2 a}$ may be written as a function of four dimensionless groups: $k_{\zeta}, k_{\lambda}, k_{\psi, 0}$ and $k_{T, a}$

$$
\begin{gathered}
R_{1 a}(i)=\frac{k_{\lambda}}{k_{\zeta}+k_{\lambda}} \\
R_{2 a}(i)=\frac{k_{\psi, 0} \cdot e^{-K_{T, a}\left(k_{\lambda}+k_{\psi, 0}\right)}-k_{\lambda} \cdot e^{-\left(k_{\lambda}+k_{\psi, 0}\right)}}{k_{\lambda}+k_{\psi, 0}}+ \\
\frac{k_{\lambda} \cdot\left[e^{-k_{T, a}\left(k_{\zeta}+k_{\lambda}+k_{\psi, 0}\right)}-e^{-\left(k_{\zeta}+k_{\lambda}+k_{\psi, 0}\right)}\right]}{k_{\zeta}+k_{\lambda}+k_{\psi, 0}}
\end{gathered}
$$


where

$$
\begin{gathered}
k_{\zeta}=\frac{\zeta V_{s}}{\varphi S} \\
k_{\lambda}=\frac{\lambda V_{s}}{i Q_{p}} \\
k_{\psi, 0}=\frac{\psi V_{s}}{Q_{0}} \\
k_{T, a}=\frac{I E T D Q_{0}}{V_{s}}
\end{gathered}
$$

Combining equations (3) and (4), the risk of overflow for a SSS with PS and above ground $\mathrm{SU}$ is:

$$
R_{a}=R_{1 a}(i)+R_{2 a}(i)
$$

Under the non-conservative assumption that the SU is empty at the onset of each rain event, the long inter-arrival time risk of $\mathrm{SU}$ overflow is:

$$
R_{2 a}^{\prime}(i)=\int_{\frac{V_{s}}{i Q_{p}}}^{\infty} f_{\tau} \int_{0}^{\frac{i Q_{p} \tau}{\varphi S}} f_{h} d h d \tau=e^{-k_{\lambda}}-\frac{k_{\lambda}}{k_{\zeta}+k_{\lambda}} e^{-\left(k_{\zeta}+k_{\lambda}\right)}
$$

Equation(10) corresponds to the lower limit of equation (4) for small $k_{\psi, 0}$ and $k_{T, a}$.

\subsection{Risk of overflow of combined PS and below-ground SU}

The PS is equipped with $n+r$ pumps each one having discharge capacity $Q_{p}$. When rain starts to fall, $n+r-i$ pumps are not working.

Examining two consecutive rain events, and assuming that the tank is full at the end of the first event, if the event inter-arrival time is: $t>V_{s}$. $\left(i Q_{p}\right)^{-1}$, the tank is empty at the beginning of the second rain event and no overflow can occur if $\varphi S h<V_{s}$. Conversely, if the inter-arrival time of two consecutive rainfall events is shorter that the draw down time of $V_{s}$, i.e.: $t<V_{s} \cdot\left(i Q_{p}\right)^{-1}$, the available storage volume at the onset of the second rainfall event is $i Q_{p} t<V_{s}$. The probability $R_{b}$ of an overflow is: 


$$
R_{b}(i)=\int_{I E T D}^{\frac{V_{s}}{i Q_{p}}} f_{t} \int_{\frac{i Q_{p} t}{\varphi S}}^{\infty} f_{h} d h d t+\int_{\frac{V_{s}}{i Q_{p}}}^{\infty} f_{t} \int_{\frac{V_{s}}{\varphi S}}^{\infty} f_{h} d h d t
$$

$R_{b}$ may be written as a function of four dimensionless groups: $k_{\zeta}, k_{\lambda}, k_{T, b}$ and $k_{\psi}$.

$$
R_{b}=\frac{k_{\psi}}{k_{\psi}+k_{\zeta}} \cdot e^{-k_{T, b}\left(k_{\psi}+k_{\zeta}\right)}+\frac{k_{\zeta}}{k_{\psi}+k_{\zeta}} \cdot e^{-\left(k_{\psi}+k_{\zeta}\right)}
$$

where

$$
k_{T, b}=\frac{I E T D i Q_{p}}{V_{s}}
$$

and

$$
k_{\psi}=\frac{\psi V_{s}}{i Q_{p}}
$$

For $Q_{p}=Q_{0} n^{-1}$,

$$
k_{\psi}=\frac{n}{i} k_{\psi, 0}
$$

Assuming that the tank is empty at the onset of each rain event, the long-inter-arrival-time limit of the risk of overflow is:

$$
R_{b}^{\prime}(i)=\int_{\frac{V_{s}}{\varphi S}}^{\infty} f_{h} d h=e^{-k_{\zeta}}
$$

Equation (16) corresponds to the lower limit of equation (12) and may be derived for $k_{\psi}<<1$.

\subsection{Risk of insufficient water quality control}

The risk of insufficient detention time, i.e. the probability that the detention time of the second of two consecutive rain events, with inter-arrival $t$, is less than the design detention time $T_{d}$, is evaluated under the assumption that the tank is full after the first of the two consecutive rain events. In this way, risk analysis of water quality control is consistent with the estimate of the overflow risk, even though the former is not conservative. The risks of insufficient water treatment are: 


$$
\begin{gathered}
R_{T_{d}, a}=\int_{I E T D}^{\frac{V_{s}}{Q_{0}}} f_{t} \int_{0}^{\frac{Q_{0}\left(T_{d}+t\right)-V_{s}}{i Q_{p}}} f_{\tau} d \tau d t+\int_{\frac{V_{s}}{Q_{0}}}^{\infty} f_{t} \int_{0}^{\frac{Q_{0} T_{d}}{i Q_{p}}} f_{\tau} d \tau d t \\
R_{T_{d}, b}=\int_{I E T D}^{\frac{V_{s}}{i Q_{p}}} f_{t} \int_{0}^{\frac{i Q_{p}\left(T_{d}+t\right)-V_{s}}{\varphi S}} f_{h} d h d t+\int_{\frac{V_{s}}{i Q_{p}}}^{\infty} f_{t} \int_{0}^{\frac{i Q_{p} T_{d}}{\varphi S}} f_{h} d h d t
\end{gathered}
$$

for SSS with above- and below-ground SU respectively.

Integration of equations (17) and (18) gives:

$$
\begin{gathered}
R_{T_{d}, a}=e^{-k_{T, a} k_{\psi 0}}-\frac{k_{\lambda} e^{-\left(k_{\lambda, T_{d}}+k_{\psi 0}\right)}+k_{\psi 0} e^{\left[-k_{\lambda, T_{d}}-k_{T, a} k_{\psi 0}-k_{\lambda}\left(k_{T, a}-1\right)\right]}}{k_{\lambda}+k_{\psi 0}} \\
R_{T_{d}, b}=e^{-k_{T, b} k_{\psi}}-\frac{k_{\zeta} e^{-\left(k_{\zeta, T_{d}}+k_{\psi}\right)}+k_{\psi} e^{\left[-k_{\zeta, T_{d}}+k_{T, b} k_{\psi}-k_{\zeta}\left(k_{T, b}-1\right)\right]}}{k_{\zeta}+k_{\psi}}
\end{gathered}
$$

where

$$
k_{\lambda, T_{d}}=\frac{\lambda Q_{0} T_{d}}{i Q_{p}}
$$

and

$$
k_{\zeta, T_{d}}=\frac{\zeta i Q_{p} T_{d}}{\varphi S}
$$

Assuming that the $\mathrm{SU}$ is empty at the beginning of each rain event, the risks of insufficient detention time for SSS with above and below ground SU become:

$$
\begin{gathered}
R_{T_{d}, a}^{\prime}=\int_{0}^{\frac{Q_{0} T_{d}}{i Q_{p}}} f_{\tau} d \tau=1-e^{-k_{\lambda, T_{d}}} \\
R_{T_{d}, b}^{\prime}=\int_{0}^{\frac{i Q_{p} T_{d}}{n \varphi S}} f_{h} d h=1-e^{-k_{\zeta, T_{d}}}
\end{gathered}
$$

